\title{
Using Elastically Actuated Legged Robots in Rough Terrain: Experiments with DLR Quadruped bert
}

\author{
Daniel Seidel \\ Technical University Munich \\ Munich, Germany, and \\ German Aerospace Center (DLR) \\ daniel.seidel@tum.de
}

\author{
Florian C. Loeffl
German Aerospace Center (DLR) \\ German Aerospace Center (DLR) \\ Wessling, Germany \\ florian.loeffl@dlr.de
}

\author{
Thomas Gumpert \\ German Aerospace Center (DLR) \\ Wessling, Germany \\ thomas.gumpert@dlr.de
}

\begin{abstract}
This paper addresses walking and balancing in rough terrain for legged locomotion in planetary exploration as an alternative to the commonly used wheeled locomotion. In contrast to the latter, where active balancing is not necessary, legged locomotion requires constant effort to keep the main body stabilized during motion. While common quadrupedal robots require to carefully plan motions through torque control and force distribution, this paper presents an approach where elastic elements in the drive train function as an intrinsic balancing component that allows to ignore inaccuracies in the locomotion pattern and passively accommodate for terrain unevenness. The approach proposes a static walking gait algorithm, which is formulated for a general quadrupedal robot, and a hardware foot design to support the locomotion pattern. The method is experimentally tested on an elastically actuated quadrupedal robot.
\end{abstract}

\section{TABle of Contents}

1. INTRODUCTION............................. 1

2. ROBOT MODEL FORMULATION ............... 2

3. Static Walking Gait ...................... 3

4. Planar foot Design......................... 4

5. EXPERIMENTS ............................ 5

6. Conclusion .............................. 5

ACKNOWLEDGMENTS .......................... 5

REFERENCES ................................. 6

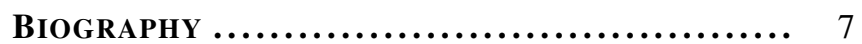

\section{INTRODUCTION}

Planetary space exploration focuses mostly on wheeled locomotion, as rover-type vehicles are able to navigate in a multitude of different terrains and soils. Using wheels allows highly energy-efficient locomotion on planar surfaces while carrying substantial payloads. However, they fall short once obstacles like small stones and boulders have to be trespassed, because the vehicle is often not able to overcome the obstacle or the condition of the soil could inflict too much material wear out. In consequence, many scientifically highly interesting locations in current missions cannot be reached. Legged locomotion systems can potentially overcome these shortcomings. They are more robust to specific ground conditions, as legs provide the capability of targeted positioning of feet to adapt to non-flat terrain. They are generally considered

978-1-7281-2734-7/20/\$31.00 (C)2020 IEEE

\author{
Alin Albu-Schäffer \\ German Aerospace Center (DLR) \\ Wessling, Germany, and \\ Technical University Munich \\ alin.albu-schaeffer@dlr.de
}

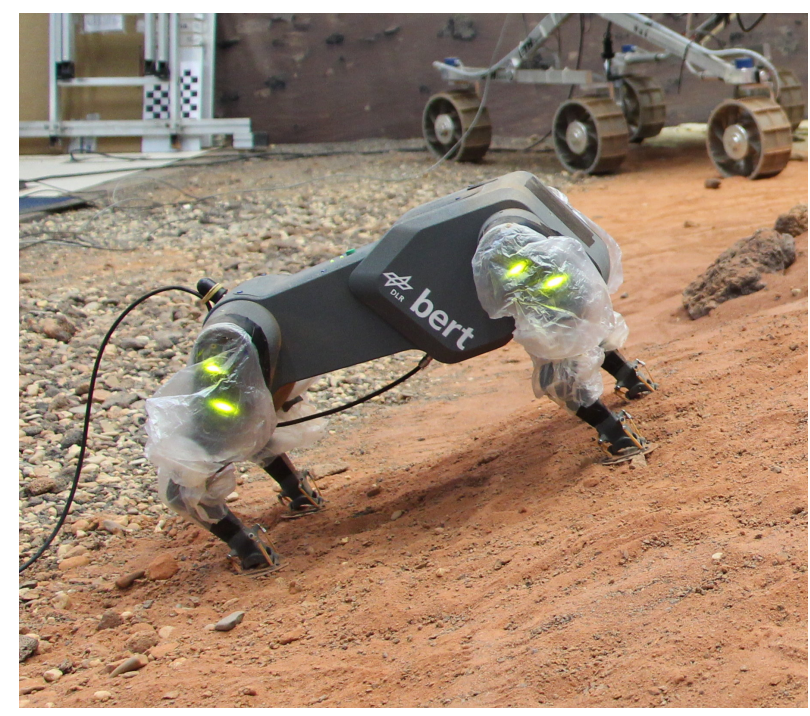

Figure 1. DLR's compliantly actuated quadruped robot bert in ESA's Planetary Robotics Lab walking up a rocky hill with prototype planar feet.

to have better mobility on uneven terrain than their wheeled and tracked counterparts because isolated footholds allow to optimize support and traction [1]. This allows to traverse and to circumvent a wider range of terrain and obstacles. Several legged systems have already shown tremendous locomotion capabilities on flat and uneven ground [2], [3], [4] . Recently, also the development of small scale quadrupedal robots has become a focus of research, examples being MIT's Mini Cheetah [5], Boston Dynamics' SpotMini [6] or Ghost Robotics' Minitaur [7]. The combination of a high payload rover for long distance travel with a small legged robot for tasks like sample collection could increase the number of reachable spots in extraterrestrial exploration.

This paper experimentally investigates the capabilities of a cat-sized quadrupedal robot with series elastic actuators (SEA) in rough terrain. The utilization of compliant actuators increases robustness of a system because elastic elements in the drive train smooth forces exerted onto the leg and therefore reduce force peaks exerted onto the motors [8], [9]. This reduces force peaks due to impacts and unexpected contact forces. Furthermore, the elastic elements in the drive 
train inherently provide the robot with adaptation capabilities. The robot can measure contact forces through the deflection of the link without the need for additional force sensors and inaccuracies of terrain perception and sensor readings can be absorbed by the springs.

Balancing and locomotion of legged robots has been an ongoing research topic for decades and a large amount of approaches focus on inverse dynamics control [10], [11], or contact force and joint torque control [12], [13], [14], [15], [16], [17], [3]. These approaches are capable of overcoming the problems of over-actuation and redundancy present in force and torque-based legged locomotion systems. However, they strictly depend on high-bandwidth joint torque interfaces. The system at hand is highly compliant and does not allow for such a high-bandwidth joint torque interface.

In previous work we proposed an analytical solution to the static balancing task [18] by formulating the problem as the task to distribute contact forces caused by gravity. The approach focused on controlling internal tensions by moving the equilibrium of intrinsic elasticities. The main goal was to maintain a constant trunk orientation during movement and adapt the leg configurations to small obstacles. However, this approach falls short once increased slopes and loose or slippery soil are approached. This paper proposes a method for walking and balancing with a compliantly actuated quadrupedal robot in uneven terrain without use of a joint torque interface. The method builds on the idea to exploit the inherent property of such a compliant legged system to rest in an equilibrium position, i. e., passively move into a balanced position when placed on the ground without the need to handle over-actuation and redundancy and without knowledge of the underlying terrain.

The target robot platform is equipped with SEAs for all joints and each leg features two degrees of freedom (DOF), a hip joint and a knee joint. Due to the lack of abduction joints the robot is not able to change its yaw angle without slip, so that this work focuses on forward movement only. The experiments consider different slopes and different terrains, namely, lava sand, fine sand, and rocky grounds. We introduce a static walking gait controller that relies solely on joint torque measurements from spring deflection and sensor readings of an inertia measurement unit (IMU) data to detect ground contact and to balance the robot during leg lift off phases. In addition, the robot has been equipped with a new foot design with a planar contact surface instead of commonly used point feet and with a passive $2 \mathrm{D}$ joint. This increased the contact surface for balancing and walking in soft soil and reduced slippage on sloped terrain. With the combination of the walking controller and the planar foot the robot is able to move on the wide range of different terrains.

\section{Robot Model Formulation}

The walking gait algorithm will be introduced for a quadruped robot model with compliantly actuated legs. The legs are attached to the trunk through the hip axis while the actuator units are located inside the trunk and connected through belt drives. Each leg consist of a hip and a knee joint with associated coordinates $q_{1}$ and $q_{2}$, respectiely. The shank is connected to the actuator via a concentric pulley such that its angle is decoupled from the hip joint and can be positioned independently relative to the main body. The knee angle, i. e., the angle between thigh and shank, can be expressed by the relative coordinate $q_{3}=q_{2}-q_{1}$. The thigh and shank

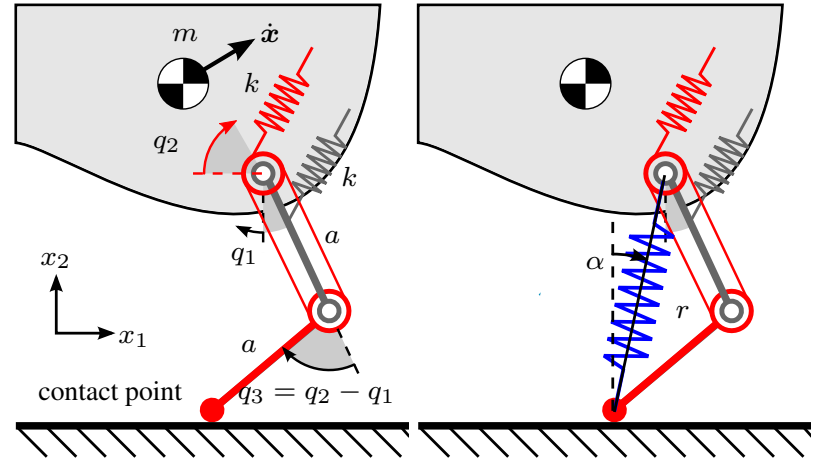

Figure 2. Model of the compliantly actuated leg (left) and description of the task coordinates (right).

segments are assumed to have equal length $a \approx 8 \mathrm{~cm}$ and their masses are assumed to be negligible compared to the mass of the trunk $m \approx 3 \mathrm{~kg}$.

Both motors are connected to the links through a linear torsional spring with constant stiffness $k \approx 1.7 \frac{\mathrm{Nm}}{\mathrm{rad}}$. For kinematic calculations the feet are considered to be point feet. However, during parts of the experiments the feet are replaced with a passive adaptive planar feet, which is explained in detail in Sec. 4. A single leg is depicted in Fig. 2 (left).

For the implementation of the walking gait, we introduce task coordinates $r$ and $\alpha$ which are the polar coordinate transformation of the joint angles. Hereby, $r$ is the total length of the leg in radial direction and $\alpha$ is the polar rest angle. They are considered as control input, i. e., the leg positions before the elastic component. We express the link positions after the spring as $\bar{r}$ and $\bar{\alpha}$. An illustration of the task coordinates is given in Fig. 2 (right).

As a result of the 2-DOF leg kinematics, the trunk has only the two translational degrees of freedom of the sagittal plane as well as rotation in roll and pitch direction. Therefore, the position of the trunk is measured by Cartesian coordinates $\boldsymbol{x} \in \mathbb{R}^{4}$. A yaw rotation is kinematically only possible to a limited degree by slippage. In consequence, for the remainder of the paper we only focus on straight-forward motion and neglect any situation that would require foot placement orthogonal to the forward direction.

Due to a lack of contact and force sensing we use the deflection of the link and motor positions to estimate the external torque applied to each joint as

$$
\tau_{i}=k\left(\theta_{i}-q_{i}\right)
$$

where $\theta_{i}$ is the motor position before the elastic component. To detect ground contacts for each leg we define the elastic force in radial direction $\tau_{r}$ as

$$
\tau_{r}=\tau_{\text {hip }}+\tau_{\text {knee }}
$$

and then use a threshold $\epsilon_{\tau_{r}}>0$ to determine if a leg is in contact with the ground. 


\section{Static Walking Gait}

In order to perform static walking and balancing, the controller applied here ensures that the projection of the robots center of mass (COM) along the gravitational acceleration stays within the horizontal plane that ensures stability, termed the support polygon. The support polygon is the horizontal region over which the center of mass must lie to achieve static stability. For a legged system, the support polygon is defined as the plane spanned by the projection of all legs with ground contact along the gravitational axis to a common horizontal plane [19]. A visualization of the support polygon is given in Fig. 3.

The walking gait is implemented as a finite state machine. For the static gait, only one leg at a time is swinging while the other three legs stay in contact with the ground. The robot follows a sequence of six actions to perform a step for one leg and then advances to the next leg. The actions are (i) COM shift, (ii) target leg unload, (iii) target leg lift, (iv) target leg swing, (v) target leg extension and (iv) counter leg extension. An overview of the state machine is given in Fig. 4. The
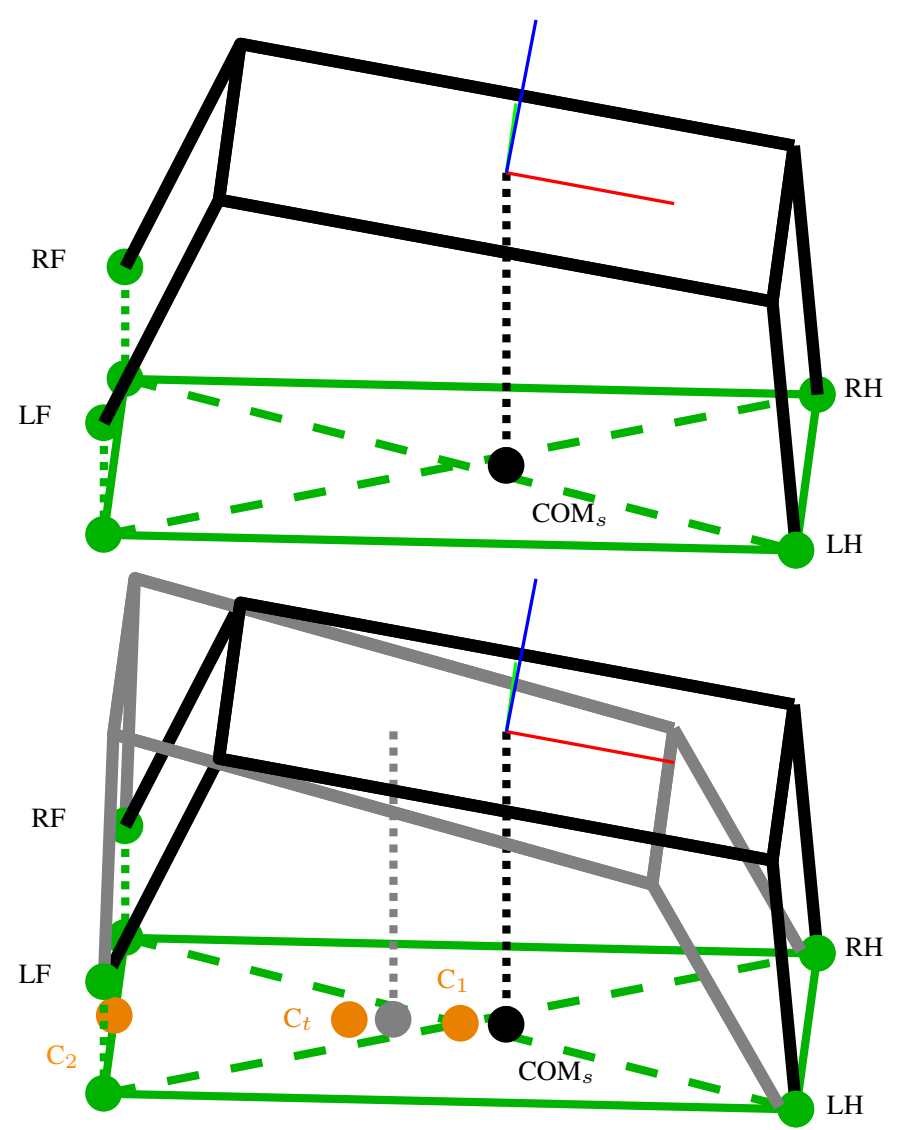

Figure 3. Support polygon of the quadruped. The top image depicts a resting position with all four legs in ground contact and the projection of the COM to the support plane (green). The bottom image shows the intersection points $C_{1}$ and $C_{2}$ of the COM projection in forward direction with the support polygon borders. $C_{t}$ is a potential target point for the

COM projection within the support polygon. The gray quadruped demonstrates an adjusted position where the COM projection is within the support polygon of LF, RF and $\mathrm{RH}$ so that LH could be lifted up.

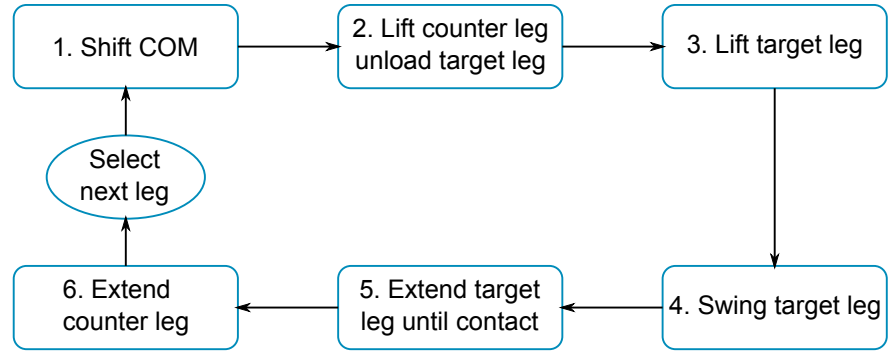

Figure 4. Walking control state machine.

order in which the legs perform the steps is predefined as: (i) right hindleg, (ii) right foreleg, (iii) left hindleg and (iv) left foreleg.

Following is a description of the individual actions, exemplified by the movement of the right foreleg. For the other three legs the leg indices have to be adjusted accordingly. The whole cycle for one leg is depicted in Fig. 5.

\section{Shift COM}

For each leg, the sequence starts with an adjustment of the center of mass. In order to be able to lift one leg without falling, the robot has to move its COM to a position where it is within the support polygon of the other three legs that will stay in contact with the ground.

As the robot is restricted to translational movement in sagittal direction only, the projection of the COM can only be shifted along a straight line in sagittal direction. Since the robot is commanded with motor positions before the elastic component and not link positions after, there is always a discrepancy between commanded motor position and the resulting link position. In consequence, it is not possible to accurately command a new COM position via inverse kinematics. Therefore, we decided to use a heuristic to determine a new configuration to adjust the COM, which is parametrizable with a single parameter which scales the shift along the sagittal direction. The value for the parameter is then tuned empirically. Let $R F_{s}, L F_{s}, R H_{s}$ and $L H_{s}$ be the projection of the foot positions on the support plane. Then, we calculate the intersection of the line extending from the $C O M_{s}$ in sagittal direction with the line between $R H_{s}$ and $L F_{s}, C_{1}$, and the line between $R H_{s}$ and $L H_{s}, C_{2}$ by solving the linear systems

$$
\mathbf{C}_{\mathbf{1}}=\left(\begin{array}{ll}
\mathbf{L F}_{\mathbf{s}}-\mathbf{R H}_{\mathbf{s}} & \mathbf{e}_{\mathbf{x}}
\end{array}\right)^{+}\left(\mathbf{C O M}_{\mathbf{s}}-\mathbf{R H}_{\mathbf{s}}\right)
$$

and

$$
\mathbf{C}_{\mathbf{2}}=\left(\begin{array}{ll}
\mathbf{L H}_{\mathbf{s}}-\mathbf{R H}_{\mathbf{s}} & \mathbf{e}_{\mathbf{x}}
\end{array}\right)^{+}\left(\mathbf{C O M}_{\mathbf{s}}-\mathbf{R H}_{\mathbf{s}}\right),
$$

where $e_{x}$ denotes the unit vector in sagittal direction and + the Moore-Penrose Pseudo Inverse. Alternatively, any other means to solve the simple equation system can be used instead. Using $C_{1}$ and $C_{2}$, we can move the target $C_{t}$ for the $\mathrm{COM}$ between the end points of the support line by simply interpolating between the two points using a shift factor $s$

$$
\mathbf{C}_{\mathbf{t}}=\mathbf{s} \cdot \mathbf{C}_{\mathbf{1}}+(\mathbf{1}-\mathbf{s}) \cdot \mathbf{C}_{\mathbf{2}} .
$$

The distance between $C O M_{s}$ and $C_{t}$ is transformed taking into account the body orientation or ground inclination

$$
D_{\text {shift }}=\frac{\left|C_{t}-C O M_{s}\right|}{\cos \left(x_{\text {pitch }}\right)},
$$



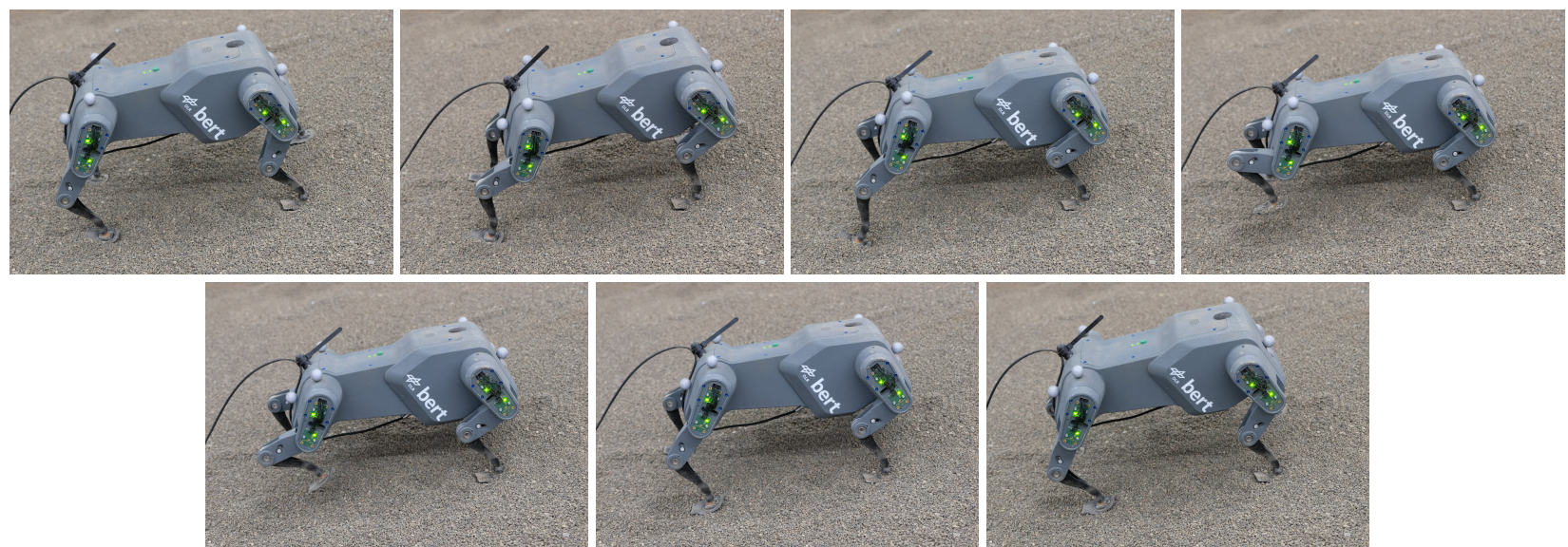

Figure 5. A complete step cycle of the state machine for one leg. From left to right and top to bottom: (i) initial position, (ii) COM shift forward, (iii) unloading main leg by retracting the counter leg and extending the target leg, (iv) lifting the target leg, (v) swinging forward, (vi) extending target leg until contact and (vii) going back to an upright position by extending counter leg to normal length.

, $x_{\text {pitch }}$ being the rotation around the $y$-axis (pitch angle), and then translated into a shifting angle

$$
\gamma=\arccos \left(1-\frac{D_{\text {shift }}^{2}}{4 r_{\text {avg }}^{2}}\right),
$$

where $r_{\text {avg }}$ denotes the average leg length of the three supporting legs. This transformation calculates the polar angle change $\gamma$ in the legs required for the trunk to move in horizontal direction by a distance $D_{\text {shift }}$. The $\gamma$ is then added as an offset to all legs.

$$
\alpha=\hat{\alpha}+\gamma
$$

\section{Lift Counter Leg and Unload Main Leg}

After shifting the COM, the robot unloads the target leg by lifting the counter leg, i.e., the diagonally opposite leg, a short distance to tilt the trunk in the direction of the counter leg. This is further supported by extending the main leg a short distance to force a tilt of the trunk in direction of the counter leg.

$$
\begin{array}{r}
r^{L H}=\hat{r}^{L H}-\Delta r_{\text {unload }} \\
r^{R F}=\hat{r}^{R F}+\Delta r_{\text {tilt }} .
\end{array}
$$

\section{Lift Target Leg}

The target leg is contracted to lift the foot off the ground

$$
r^{R F}=\hat{r}^{R F}-\Delta r_{l i f t}
$$

\section{Swing Target Leg}

The swing motion for the leg is initiated by commanding a target leg angle. This angle is predefined and does not depend on the current position of the leg

$$
\alpha^{R F}=\alpha_{0}+\Delta \alpha_{\text {swing }}
$$

\section{Extend target leg until contact}

Once the leg has reached the target angle, it is commanded to extend to the predefined default length $r_{0}$. If a contact is detected during extension, the motion is stopped and the current length will be maintained until the leg steps again

$$
r^{R F}= \begin{cases}\hat{r}^{R F} & \tau_{z}^{R F}>\epsilon_{\tau_{r}} \\ r_{0} & \text { otherwise }\end{cases}
$$

\section{Extend Counter Leg}

Finally, the counter leg is commanded to extend back to its previous length

$$
r^{L H}=\hat{r}^{L H}+\Delta r_{\text {unload }} .
$$

Once the extension motion is finished, the target leg is incremented and the sequence starts from the beginning with the shift of the COM.

\section{Planar Foot Design}

For flat and clean surfaces the robot used in experiments described in the following section is equipped with rubber coated point feet. This point feet showed significant problems with slippage and burying into the soil during preliminary tests on terrain with rigid rocky surfaces covered with thin dust layers as well as in sandy terrain. To counter these shortcomings, we have implemented a special foot design that is illustrated in Fig. 6.

In order to prevent that the foot sinks into the soil the area of contact has been increased from an (almost) point contact to a planar area. In addition, analogue to shoe soles the bottom of the contact area has been equipped with an uneven profile with the target to compress the soil under the foot to reduce slippage. However, as the angle between the legs and the surfaces changes during motion this would result in motions where only the boundaries of the contact areas would be in contact with the ground. This necessitates the use of a passive-compliant joint in the form of an elastic silicone tube between the end of the shank and the foot plate, which 


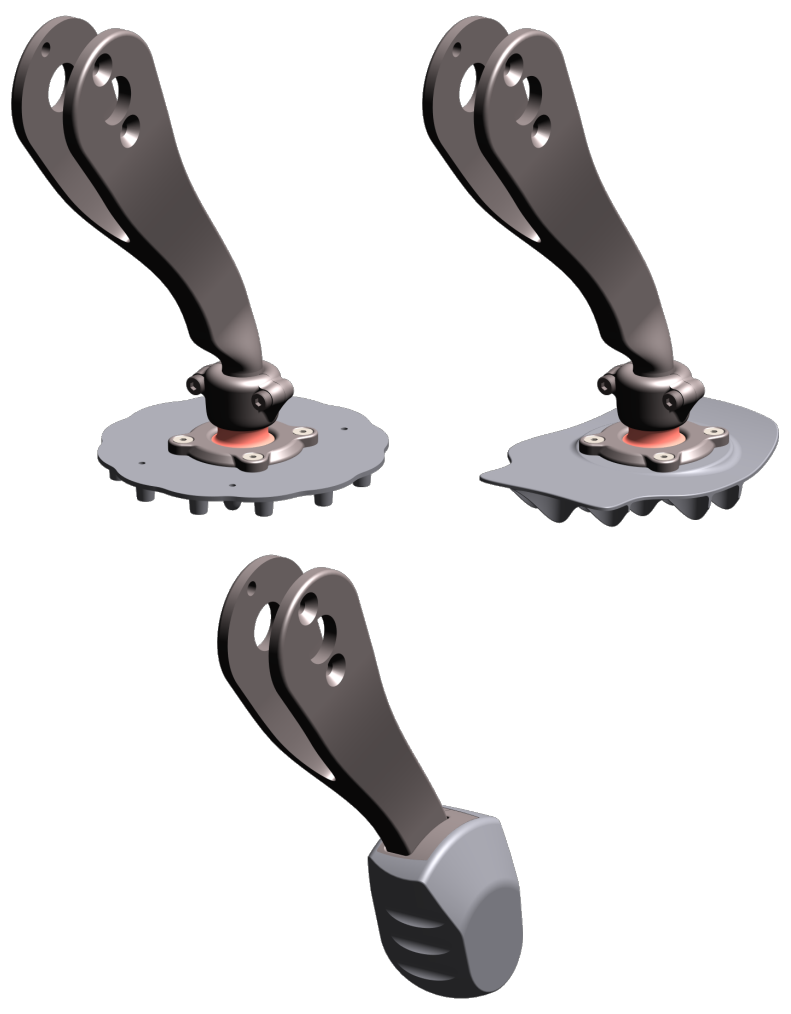

Figure 6. CAD model of the different feet. The upper two feet use a passive planar joint with different contact plate designs. The first contact plate is circular shaped and equipped with simple spikes (top left), the second plate features a pattern of small shovels at the bottom and a forward displaced force transmission point (top right). The foot displayed at the bottom is the regular rubber point foot normally used for normal conditions on flat surface.

allows the foot to automatically adapt to the angle between the contact area and the ground.

For the foot plate itself, we implemented two different versions, one similar to spiked shoes with a circular surface centered around the joint (Fig. 6, top left) and one asymmetrical surface (Fig. 6, top right) with a shovel like pattern directed along the movement direction. Further, for the asymmetrical plate the mount point, i. e., the point where the joint is connected to the plate, has its force transmission point displaced to the front. This was designed to passively imitate the behavior of humans and animals tilting their feet forward to compress the underlying soil to a support platform. This effect was visually verified during experiments.

\section{EXPERIMENTS}

The static walking algorithm has been tested experimentally on DLR's compliantly actuated quadruped robot platform bert, which is described in detail in [20]. The robot is equipped with low-cost off-the-shelf servo drives, which are commanded through position control. In the following, the proposed walking algorithm will be experimentally verified and the feasibility of compliant actuation for uneven terrain demonstrated.

Considering the concept of the stability margin ${ }^{2}$ from Smith Weinstein [19] results in situations that make it necessary to shift the COM backwards, i.e., against the locomotion direction, to be able to lift the forefeet. This is especially difficult when traversing high slopes, where the body quickly loses foothold and balance due to the inclination.

For the experiments the robot was commanded to traverse along a straight path in a lava sand bed. The bed features a ramp with adjustable inclination and all three feet designs described in Sec. 4 were tested with $0^{\circ}, 10^{\circ}, 15^{\circ}, 20^{\circ}$, $22.5^{\circ}, 25^{\circ}, 27.5^{\circ}$ and $30^{\circ}$ inclination. To ensure equal conditions, the bed was leveled before each trial. The robot was placed in a horizontal position in front of the ramp and then commanded to climb up the chosen slope. The results of the experiments are depicted in Fig. 7. In general, the robot was able to climb up inclinations up to $27.5^{\circ}$ and reached its limit at $30^{\circ}$, where slippage and the inability to balance the COM over the support polygon led to down-sliding and falls.

When comparing the three feet designs, we notice that there was no substantial difference for inclinations up to $15^{\circ}$ with the exception that the point foot was performing minimally faster than the two joint-based feet. At $20^{\circ}$ and upwards the circular spiked foot started to suffer from increased slippage, while the point foot was still able to provide a secure foothold. Steeper inclinations caused the circular spiked foot to stop gaining height at $22.5^{\circ}$. From an inclination of $25^{\circ}$ on, both the point foot and the circular spiked foot were no longer able to reach the finishing point. The second planar foot was able to provide enough foothold to move up the slope with $27.5^{\circ}$ inclination, but ultimately failed at $30^{\circ}$ at the beginning of the slope.

During experiments it could be observed that for higher inclinations the robot frequently reached joint limits, which prevented sufficient unloading of the swing leg. This aspect will be considered in later design. Nevertheless, despite the inability to balance the COM in lateral direction it was possible to overcome highly inclined and slippery terrain, which is difficult to traverse even for humans.

\section{Conclusion}

This paper has demonstrated great locomotion capabilities of a quadrupedal robot in rough terrain through exploitation of intrinsic passive compliance. The serial elastic actuation in combination with a static balancing gait is able to traverse highly inclined and slippery terrain without relying on highbandwidth torque control or sophisticated force distribution. A special foot design with a passive adaptive planar joint further increased the capability of the system to adapt to the terrain. Future work will focus on implementing the shoulder joint and advancing the locomotion capabilities to allow navigating around obstacles and increase the magnitude of slopes and surface irregularities the robot is able to overcome.

\section{ACKNOWLEDGMENTS}

This work was partially conducted at the European Space Research and Technology Centre (ESTEC) of the European

${ }^{2}$ The stability margin is defined as the minimum distance of the projection of the COM along the gravitational axis to the boundaries of the support polygon. 

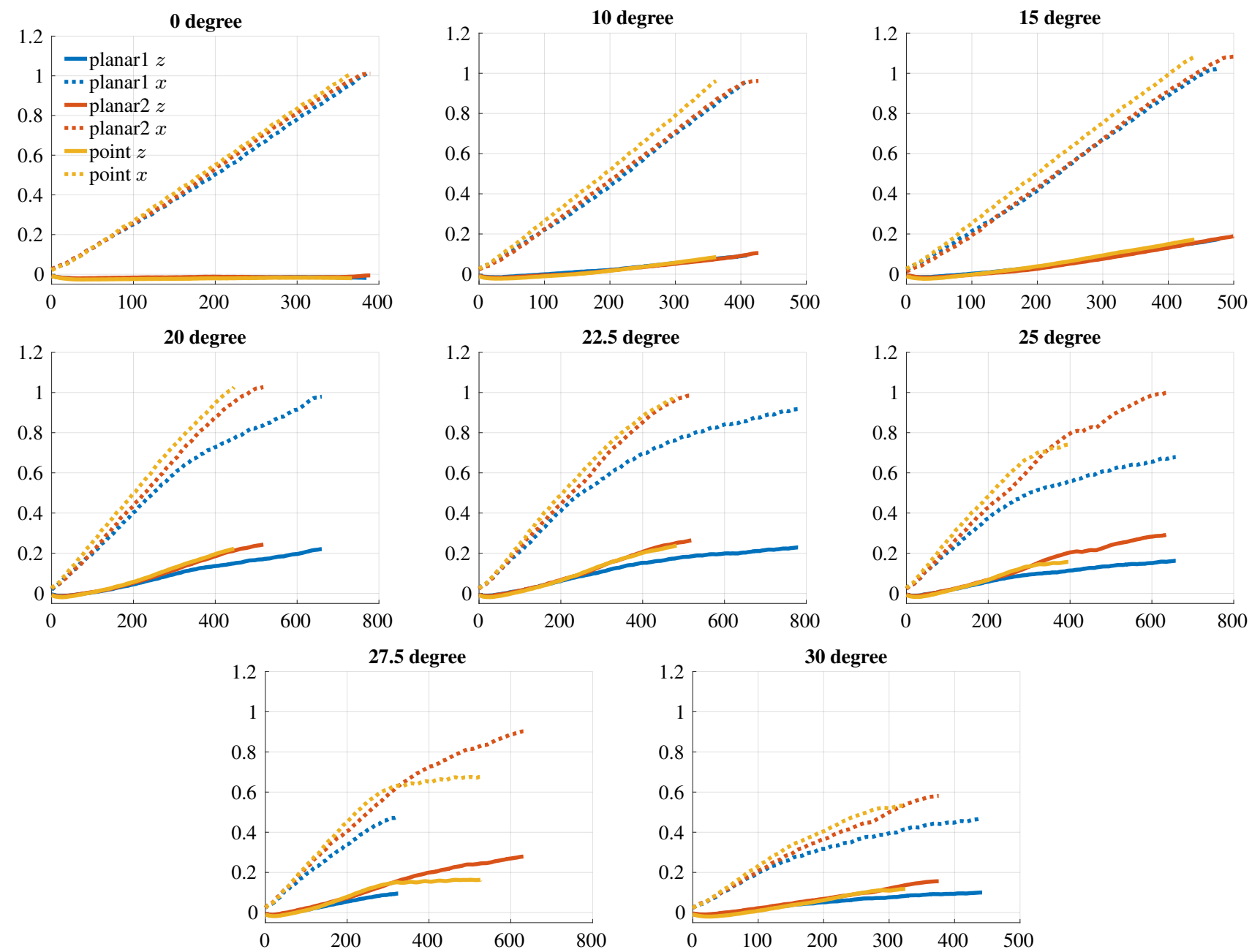

Figure 7. Experimental results of approaching lava sand terrain with slopes ranging from $0^{\circ}$ to $30^{\circ}$. Three different feet were compared against each other. The plots show for each foot both the horizontal and vertical movement progress [m], $x$ and $z$, on the vertical axes versus time [s] on the horizontal axis.

Space Agency with support of Gianfranco Visentin within the M-Runner Network/Partnering Initiative Programme.

\section{REFERENCES}

[1] M. H. Raibert, Legged Robots That Balance. The MIT Press, 1986.

[2] M. Raibert, K. Blankespoor, G. Nelson, and R. Playter, "Bigdog, the rough-terrain quadruped robot," IFAC Proceedings Volumes, vol. 41, no. 2, pp. 10822 - 10825 , 2008, 17th IFAC World Congress.

[3] G. Bledt, M. J. Powell, B. Katz, J. Di Carlo, P. M. Wensing, and S. Kim, "Mit cheetah 3: Design and control of a robust, dynamic quadruped robot," in 2018 IEEE/RSJ International Conference on Intelligent Robots and Systems (IROS), Oct 2018, pp. 2245-2252.

[4] C. Semini, N. G. Tsagarakis, E. Guglielmino, M. Focchi, F. Cannella, and D. G. Caldwell, "Design of hyq-a hydraulically and electrically actuated quadruped robot," Proceedings of the Institution of Mechanical Engineers, Part I: Journal of Systems and Control En- gineering, vol. 225, no. 6, pp. 831-849, 2011.

[5] B. Katz, J. D. Carlo, and S. Kim, "Mini cheetah: A platform for pushing the limits of dynamic quadruped control," in 2019 International Conference on Robotics and Automation (ICRA), May 2019, pp. 6295-6301.

[6] E. Ackerman, "Boston dynamics' spotmini is all electric, agile, and has a capable facearm," IEEE Spectrum, 10 2016. [Online]. Available: http://spectrum.iee.org/automaton/robotics/ home-robots/boston-dynamicsspotmini

[7] _ - "Ghost robotics' minitaur quadruped conquers stairs, doors, and fences and is somehow affordable," IEEE Spectrum, 10 2016. [Online]. Available: http://spectrum.ieee.org/automaton/robotics/ roboticshardware/ghost-robotics-minitaur-quadruped

[8] R. M. Alexander, "Three uses for springs in legged locomotion," International Journal of Robotics Research, vol. 9, no. 2, pp. 53-61, 1990.

[9] G. A. Pratt and M. M. Williamson, "Series elastic actuators," in Intelligent Robots and Systems 95.'Human Robot Interaction and Cooperative Robots', Pro- 
ceedings. 1995 IEEE/RSJ International Conference on, vol. 1. IEEE, 1995, pp. 399-406.

[10] M. Mistry, J. Buchli, and S. Schaal, "Inverse dynamics control of floating base systems using orthogonal decomposition," in ICRA, May 2010, pp. 3406 - 3412.

[11] L. Righetti, J. Buchli, M. Mistry, M. Kalakrishnan, and S. Schaal, "Optimal distribution of contact forces with inverse dynamics control," IJRR, vol. 32, no. 3, pp. 280 $-298,2013$.

[12] C. Gehring, S. Coros, M. Hutter, M. Blösch, M. Hoepflinger, and R. Siegwart, "Control of dynamic gaits for a quadrupedal robot," in IEEE Int. Conf. on Robotics and Automation, May 2013, pp. 3287-3292.

[13] M. Hutter, C. Gehring, M. A. Höpflinger, M. Blösch, and R. Siegwart, "Toward combining speed, efficiency, versatility, and robustness in an autonomous quadruped." IEEE Trans. Robotics, vol. 30, no. 6, pp. 1427-1440, 2014.

[14] L. Sentis, "Compliant control of whole-body multicontact behaviors in humanoid robots," in Motion Planning for Humanoid Robots, K. Harada, E. Yoshida, and K. Yokoi, Eds. Springer London, 2010, pp. 29-66.

[15] P. M. Wensing, G. B. Hammam, B. Dariush, and D. E. Orin, "Optimizing foot centers of pressure through force distribution in a humanoid robot," International Journal of Humanoid Robotics, vol. 10, no. 03, pp. 1-21, 2013.

[16] B. Henze, A. Dietrich, and C. Ott, "An approach to combine balancing with hierarchical whole-body control for legged humanoid robots," RAL, vol. 1, no. 2, pp. $700-$ 707, July 2016, date of Publication: 29 December 2015.

[17] B. Henze, M. A. Roa, and C. Ott, "Passivity-based whole-body balancing for torque-controlled humanoid robots in multi-contact scenarios," IJRR, vol. 35 , no. 12 , pp. $1522-1543,2016$.

[18] D. Lakatos, Y. Federigi, T. Gumpert, B. Henze, M. Hermann, F. Loeffl, F. Schmidt, D. Seidel, and A. AlbuSchäffer, "A coordinate-based approach for static balancing and walking control of compliantly actuated legged robots," in 2019 International Conference on Robotics and Automation (ICRA), May 2019, pp. 95099515.

[19] R. McGhee and A. Frank, "On the stability properties of quadruped creeping gaits," Mathematical Biosciences, vol. 3, pp. $331-351,1968$.

[20] D. Lakatos, K. Ploeger, F. Loeffl, D. Seidel, F. Schmidt, T. Gumpert, F. John, T. Bertram, and A. Albu-Schäffer, "Dynamic locomotion gaits of a compliantly actuated quadruped with slip-like articulated legs embodied in the mechanical design," IEEE Robotics and Automation Letters, vol. 3, no. 4, pp. 3908-3915, 2018.

\section{BIOGRAPHY}

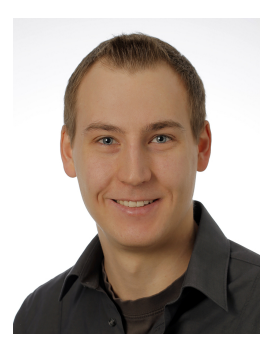

Daniel Seidel received his M.Sc. degree in "Intelligent Systems" from Bielefeld University, Germany, in 2014. Afterwards he joined the Chair for Sensor Based Robotic Systems and Intelligent Assistance Systems of Prof. AlbuSchäffer at the Technical University of Munich. Futhermore, he is an ongoing researcher at the Institute of Robotics and Mechatronics at the German Aerospace Center (DLR) where he is involved in the development of the DLR quadruped robot bert. His main interests lie in motion generation and planning for elastically actuated legged robots and machine learning on hardware systems.

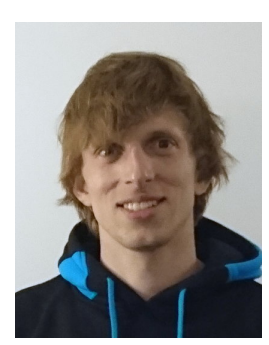

Milan Hermann received his diploma degree in aerospace engineering from University of Stuttgart in 2017. He joined the German Aerospace Center (DLR) Institute of Robotics and Mechatronics in 2016, where he was involved in developing the flexible wing structure for a high-altitude aircraft. His main interests lie in lightweight construction, elastic structures, composites manufacturing and rapid prototyping. At this time he is concentrating on mechanical design of a small quadruped robot for the investigation of dynamic legged locomotion.

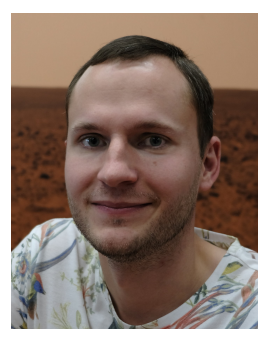

Thomas Gumpert received his B.Eng. in Mechatronics 2012 and in 2018 his M.Sc. in Applied Research on Mechatronic Systems from the University of Applied Sciences Augsburg. He joined the German Aerospace Center (DLR) Institute of Robotics and Mechatronics in 2008 with focus on electrical drives. Since 2015 he is heading the Drive Technology Lab. As part of different teams he was involved in the development of the lightweight robot SARA and space qualified robotic arm CAESAR as well as the space qualified force feedback joystick Kontur-2. Currently he is responsible for the robotic hardware of the humanoid robots Rollin and Agile Justin and he coordinates the development of DLR's quadruped robot bert with his main focus on actuator and sensor electronics.

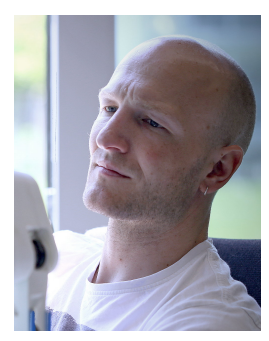

Florian Loeff received his M.Sc. Degree in Mechanical Engineering from Technical University of Munich, Germany, in 2008. He joined the German Aerospace Center (DLR) in 2014 as leader of the development team of the elastic bipedal robot C-Runner. Then he joined the development team of the quadruped bert. He is currently head of the systems design group at DLRs Institute of Robotics and Mechatronics. He supported the bert development as systems engineer and mechanical engineer. 


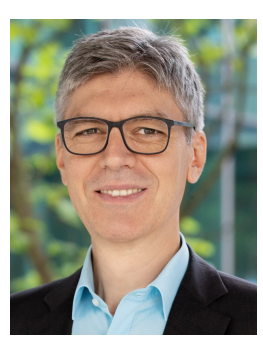

Alin Albu-Schäffer Alin Albu-Schäffer received his M.S. in electrical engineering from the Technical University of Timisoara, Romania in 1993 and his Ph.D. in automatic control from the Technical University of Munich in 2002. Since 2012 he is the head of the Institute of Robotics and Mechatronics at the German Aerospace Center (DLR), which he joined in 1995 as a Ph.D. candidate. Moreover, he is a professor at the Technical University of Munich, holding the Chair for "Sensor Based Robotic Systems and Intelligent Assistance Systems" at the Computer Science Department. His personal research interests include robot design, modeling and control, nonlinear control, flexible joint and variable compliance robots, impedance and force control, physical human-robot interaction, bio-inspired robot design and control. He received several awards, including the IEEE King-Sun Fu Best Paper Award of the Transactions on Robotics in 2012 and 2014; several ICRA and IROS Best Paper Awards as well as the DLR Science Award. He was strongly involved in the development of the DLR light-weight robot and its commercialization through technology transfer to $K U K A$. 